\title{
Excited states in the neutron-rich nucleus ${ }^{25} \mathbf{F}$
}

\author{
Zs. Vajta, ${ }^{1}$ M. Stanoiu, ${ }^{2}$ D. Sohler, ${ }^{1}$ G. R. Jansen, ${ }^{3,4}$ F. Azaiez,${ }^{5}$ Zs. Dombrádi, ${ }^{1}$ O. Sorlin,${ }^{6}$ B. A. Brown, ${ }^{7}$ M. Belleguic, \\ C. Borcea, ${ }^{2}$ C. Bourgeois, ${ }^{5}$ Z. Dlouhy,${ }^{8}$ Z. Elekes, ${ }^{1}$ Zs. Fülöp, ${ }^{1}$ S. Grévy, ${ }^{9}$ D. Guillemaud-Mueller, ${ }^{5}$ G. Hagen, ${ }^{4,3}$ \\ M. Hjorth-Jensen, ${ }^{7,10}$ F. Ibrahim, ${ }^{5}$ A. Kerek, ${ }^{11}$ A. Krasznahorkay, ${ }^{1}$ M. Lewitowicz, ${ }^{6}$ S. M. Lukyanov, ${ }^{12}$ S. Mandal, ${ }^{13}$ \\ P. Mayet, ${ }^{13}$ J. Mrázek, ${ }^{8}$ F. Negoita, ${ }^{2}$ Yu.-E. Penionzhkevich, ${ }^{12}$ Zs. Podolyák,,${ }^{14}$ P. Roussel-Chomaz,${ }^{15}$ M. G. Saint-Laurent,${ }^{6}$ \\ H. Savajols, ${ }^{6}$ G. Sletten, ${ }^{16}$ J. Timár, ${ }^{1}$ C. Timis, ${ }^{2}$ and A. Yamamoto ${ }^{14}$ \\ ${ }^{1}$ Institute for Nuclear Research, Hungarian Academy of Sciences, P.O. Box 51, Debrecen, H-4001, Hungary \\ ${ }^{2}$ IFIN-HH, P. O. Box MG-6, 76900 Bucharest-Magurele, Romania \\ ${ }^{3}$ Department of Physics and Astronomy, University of Tennessee, Knoxville, Tennessee 37996, USA \\ ${ }^{4}$ Physics Division, Oak Ridge National Laboratory, Oak Ridge, Tennessee 37831, USA \\ ${ }^{5}$ Institut de Physique Nucléaire, IN2P3-CNRS, F-91406 Orsay Cedex, France \\ ${ }^{6}$ Grand Accélérateur National d'Ions Lourds, CEA/DSM-CNRS/IN2P3, B.P. 55027, F-14076 Caen Cedex 5, France \\ ${ }^{7}$ National Superconducting Cyclotron Laboratory and Department of Physics and Astronomy, \\ Michigan State University, East Lansing, Michigan 48824-1321, USA \\ ${ }^{8}$ Nuclear Physics Institute, AS CR, CZ 25068, Rez, Czech Republic \\ ${ }^{9}$ CENBG, UMR 5797 CNRS/IN2P3, B. P. 120, F-33175 Gradignan Cedex, France \\ ${ }^{10}$ Department of Physics and Center of Mathematics for Applications, University of Oslo, N-0316 Oslo, Norway \\ ${ }^{11}$ Department of Physics, Royal Institute of Technology, SE-10691 Stockholm, Sweden \\ ${ }^{12}$ FLNR, JINR, 141980 Dubna, Moscow Region, Russia \\ ${ }^{13}$ Gesellschaft für Schwerionenforschung, D-64291 Darmstadt, Germany \\ ${ }^{14}$ Department of Physics, University of Surrey, Guildford GU2 7XH, United Kingdom \\ ${ }^{15}$ DSM/IRFU, CEA, F-91191 Gif sur Yvette Cedex, France \\ ${ }^{16}$ Niels Bohr Institute, University of Copenhagen, 2100 Copenhagen, Denmark
}

(Received 30 December 2013; revised manuscript received 8 May 2014; published 30 May 2014)

\begin{abstract}
The structure of the nucleus ${ }_{9}^{25} \mathrm{~F}$ was investigated through in-beam $\gamma$-ray spectroscopy of the fragmentation of ${ }^{26} \mathrm{Ne}$ and ${ }^{27,28} \mathrm{Na}$ ion beams. Based on the particle- $\gamma$ and particle- $\gamma \gamma$ coincidence data, a level scheme was constructed and compared with shell model and coupled-cluster calculations. Some of the observed states were interpreted as quasi-single-particle states built on top of the closed-shell nucleus ${ }^{24} \mathrm{O}$, while the others were described as states arising from coupling of a single proton to the $2^{+}$core excitation of ${ }^{24} \mathrm{O}$.
\end{abstract}

DOI: 10.1103/PhysRevC.89.054323

PACS number(s): 23.20.Lv, 25.70.Mn, 27.30.+t, 21.60.Cs

\section{INTRODUCTION}

Nuclei in the vicinity of doubly closed-shell nuclei are expected to exhibit simple shell model structures, which can often be interpreted in terms of selected single-particle degrees of freedom built on a closed-shell core. For stable nuclei, several such cases are known in the vicinity of, for example, nuclei like ${ }^{16} \mathrm{O},{ }^{40} \mathrm{Ca},{ }^{56} \mathrm{Ni},{ }^{132} \mathrm{Sn}$, and ${ }^{208} \mathrm{~Pb}$ that show such characteristics which are relatively well understood. However, more complex structures were observed for shortlived isotopes with extreme $N / Z$ ratios.

Recent experiments indicated that $N=16$ was a magic number close to the neutron drip line. This was first evidenced by neutron separation energy and cross section measurements [1]. Additional information came from the spectroscopy of the $N=16$ isotones. The energy spectrum of ${ }^{26} \mathrm{Ne}$ resembled that of a vibrator nucleus [2], while only unbound excited states above the one-neutron spin density $S_{n}=4.09(10) \mathrm{MeV}$ [3], namely, a spin-parity $J^{\pi}=2^{+}$state at $4.7 \mathrm{MeV}$ and a $J^{\pi}=1^{+}$at $5.3 \mathrm{MeV}$ [4,5], were found in ${ }^{24} \mathrm{O}$. Moreover, a small quadrupole deformation parameter, $\beta_{2}=0.15$ [5], as well as a large $s$ wave spectroscopic factor of $1.74 \pm 0.19$ [6] was determined for the ground state of ${ }^{24} \mathrm{O}$, providing further support for its closed $N=16$ neutron shell. It was also proven that the drip line was reached at ${ }^{24} \mathrm{O}$ for the oxygen isotopes reflected in the neutron separation energy step of $4.8 \mathrm{MeV}$ between ${ }^{24} \mathrm{O}$ and ${ }^{25} \mathrm{O}$ [7]. A compatible value of $4.95 \mathrm{MeV}$ was obtained from the $2 J+1$ (where $J$ is the spin) weighted average energy of the two $J^{\pi}=2^{+}$and $J^{\pi}=1^{+}$ states in ${ }^{24} \mathrm{O}$ [4].

Adding a single valence proton to the doubly closed-shell nucleus ${ }^{24} \mathrm{O},{ }^{25} \mathrm{~F}$ is expected to have a rather simple structure. Its energy spectrum can be described up to the neutron separation energy of 4.36(12) $\mathrm{MeV}$ [8] as a few single-proton states coupled to the ground and first excited states of ${ }^{24} \mathrm{O}$. Deviations from this straightforward picture may arise from the following: ${ }^{25} \mathrm{~F}$ is expected to be located at the frontier of emerging new structures induced by intruder configurations, possibly leading to cluster configurations at low excitation energy. Recent cluster-model calculations [9] showed that the energy of cluster states associated with proton cross shell excitations increased with increasing neutron number among the fluorine isotopes. Conversely, the energy of a new class of intruder cluster configurations associated with coherent proton and neutron cross shell excitations decreased when approaching the neutron drip line. According to these calculations all the intruder states appeared above the neutron separation energy in ${ }^{25} \mathrm{~F}$ [9]. The lowest-lying proton intruder state is predicted by shell model calculations using the Warburton-Brown psd-shell 
(WBP) interaction in the $s p s d p f$ space at about $4.3 \mathrm{MeV}$ in the ${ }^{25} \mathrm{~F}$ nucleus [10,11]. Recently, two neutron-unbound states have been reported at 28(4) [10] and 300(170) [11] above the neutron separation energy which are expected to have intruder $J^{\pi}=1 / 2^{-}$and intruder $J^{\pi}=3 / 2^{-}$or normal $J^{\pi}=5 / 2^{+}$ character, respectively.

The coupling of the weakly bound states to the continuum might also perturb the structure of ${ }^{25} \mathrm{~F}$. All the states arising from the coupling of a proton to the excited states of ${ }^{24} \mathrm{O}$ are expected to lie close to the neutron separation energy. The first excited state of ${ }^{24} \mathrm{O}$ itself is unbound by about $1 \mathrm{MeV}$ [5]. The effects of continuum coupling were shown in the description of the properties of neutron-rich oxygen isotopes in Refs. [12,13]. Coupling to the nonresonant continuum and/or to eventual lowlying resonances may also play an important role in explaining the excitation energy spectrum of ${ }^{25} \mathrm{~F}$.

Preliminary papers on the study of ${ }^{25} \mathrm{~F}$ from the fragmentation of the stable ${ }^{36} \mathrm{~S}$ beam at the Grand Accelerateur National d'Ions Lourds (GANIL) were already published [14-16]. We reported on four $\gamma$ lines, two of which were confirmed in an experiment performed at Rikagaku Kenkyusho (The Institute of Physical and Chemical Research) of Japan (RIKEN) [17]. In the final analysis of the spectra seven $\gamma$ rays could be assigned to ${ }^{25} \mathrm{~F}$ [18]; however, $\gamma-\gamma$ coincidence was not available and only a tentative level scheme could be constructed for ${ }^{25} \mathrm{~F}$ from these data. In the present paper we show the results obtained on ${ }^{25} \mathrm{~F}$ at GANIL by a detailed in-beam $\gamma$-spectroscopic study via double-step fragmentation reaction. The experimental analyzes are interpreted in terms of both shell-model and coupled-cluster (CC) calculations. The effective Hilbert space for the shell-model calculations is defined by the $1 s 0 d$ shell. $\mathrm{CC}$ calculations involve much larger effective Hilbert spaces, typically ten or more major oscillator shells. For states close to the separation energy, one expects that correlations from states in the continuum may play a larger role, suggesting, thereby, the need for larger Hilbert spaces. The larger dimensionalities mean, however, that only selected correlations are summed to infinite order in $\mathrm{CC}$ approaches, in contrast to configuration interaction calculations performed by the nuclear shell-model. For the latter, the many-nucleon eigenvalue problem is solved numerically exactly in a limited space. The degrees of freedom, of say the $1 s 0 d$ shell studied here, may however not capture the relevant physics of more neutron-rich fluorine isotopes. These aspects will be discussed in our theoretical analysis of the experimental data.

\section{EXPERIMENTAL METHODS}

In the double-step fragmentation reaction a primary beam of ${ }^{36} \mathrm{~S}$ at $77.5 \mathrm{MeV}$ A with a mean intensity of $400 \mathrm{pnA}$ was delivered by the two GANIL cyclotrons to induce fragmentation reactions in a carbon target of $348 \mathrm{mg} / \mathrm{cm}^{2}$ thickness placed in the Superconducting Intense Source for Secondary Ions (SISSI) [19] device. The produced nuclei were selected by means of the ALPHA spectrometer equipped with a $130 \mathrm{mg} / \mathrm{cm}^{2} \mathrm{Al}$ wedge at the dispersive focal plane. The magnetic rigidity of the spectrometer and the optics of the beam line were optimized for the transmission of secondary beam particles with $N / Z \approx 5 / 3$ composed of ${ }^{24} \mathrm{~F},{ }^{25,26} \mathrm{Ne}$,
${ }^{27,28} \mathrm{Na}$, and ${ }^{29,30} \mathrm{Mg}$ nuclei with energies varying from 54 to $65 \mathrm{MeV} \mathrm{A}$. These nuclei subsequently impinged on an "active" target, made of a plastic scintillator $\left(103 \mathrm{mg} / \mathrm{cm}^{2}\right)$ sandwiched by two carbon foils of $51 \mathrm{mg} / \mathrm{cm}^{2}$ each, placed at the entrance of the SPEG spectrometer [20] to induce a secondary reaction. The plastic scintillator part of the active target was used to identify the incoming nuclei through energy loss and time-of-flight measurements. A total of $3 \times 10^{4}{ }^{25} \mathrm{~F}$ nuclei were produced in the fragmentation reaction, mainly from ${ }^{26} \mathrm{Ne}$ and ${ }^{27,28} \mathrm{Na}$ secondary beams. The ${ }^{25} \mathrm{~F}$ nuclei were selected by the SPEG spectrometer and identified at its focal plane by a combined use of energy loss, total energy, time-of-flight, and focal-plane position information. This latter parameter served to correct the time-of-flight value from the various flight path lengths of the fragments in the spectrometer operated in a dispersive mode. The energy losses and positions of the fragments were determined by the use of an ionization (70 cm long) chamber and a set of two $x-y$ drift chambers ( $2 \mathrm{~cm}$ thick and $80 \mathrm{~cm}$ wide each). The residual energy of the fragments was measured in a $2 \mathrm{~cm}$ thick plastic scintillator whose timing signal served to determine the time-of-flight $\mathrm{T}_{p l}$ with respect to the cyclotron radio frequency and to the plastic scintillator of the active target.

An array of $74 \mathrm{BaF}_{2}$ crystals was mounted in two hemispheres around the active target at a mean distance of $21 \mathrm{~cm}$, covering a total solid angle of $80 \%$. It was used to detect in-flight $\gamma$ rays emitted with $v / c \simeq 0.34$ in coincidence with the ${ }^{25} \mathrm{~F}$ fragments. The $\gamma$ spectra were corrected for the Doppler-shift, yielding a full width at half maximum of about $12 \%$. The detection limit was set below $100 \mathrm{keV}$ for each detector in the laboratory frame. The efficiency of the $\mathrm{BaF}_{2}$ array was determined by a GEANT4 simulation, which was calibrated using ${ }^{137} \mathrm{Cs}$ and ${ }^{60} \mathrm{Co}$ standard $\gamma$ sources placed at the target position. A typical photopeak efficiency of about $20 \%$ is obtained at $1.3 \mathrm{MeV}$. Owing to the compact geometry of the array, high energy $\gamma$ rays could scatter from one detector to another. To reduce the background and to achieve a higher efficiency at high energies, an add-back algorithm was used for the events where at least two neighboring detectors fired at the same time. For the proper determination of the efficiency, the source and simulated spectra were also analyzed using the add-back algorithm.

\section{EXPERIMENTAL RESULTS}

The Doppler-corrected $\gamma$-ray spectrum obtained for the ${ }^{25} \mathrm{~F}$ nucleus is shown in Fig. 1. Two peaks can be seen at 750(30) and $1720(15) \mathrm{keV}$, while a broader structure including several $\gamma$ lines is present between 3 and $4.5 \mathrm{MeV}$. This observation is in agreement with the results obtained in the fragmentation of the ${ }^{36} \mathrm{~S}$ beam $[14-16,18]$.

The decomposition of the broad structure into individual peaks requires the determination of the energy-dependent $\gamma$ width of the peaks, which was obtained from single $\gamma$ peaks observed in other reaction channels in the same experiment. An almost linear energy dependence of the peak width was observed. The response function of the $\mathrm{BaF}_{2}$ crystals leads to a $\gamma$ peak with a low-energy tail, the energy-dependent shape of which was simulated by use of the GEANT4 package. This 


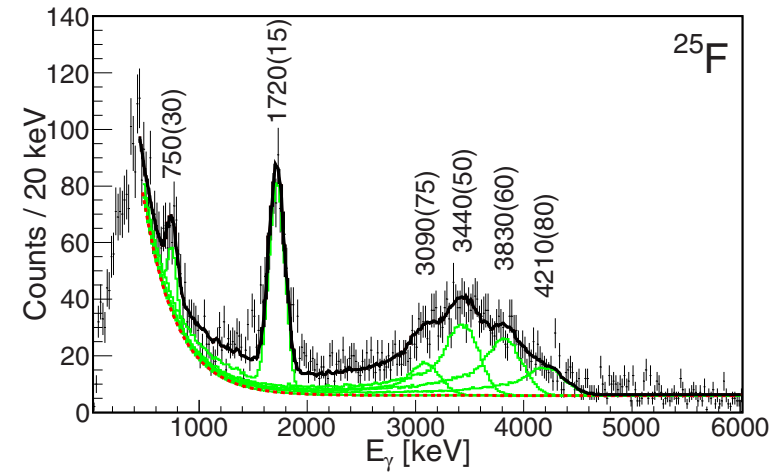

FIG. 1. (Color online) Decomposition of the $\gamma$-ray spectrum of ${ }^{25} \mathrm{~F}$. The solid black (dark) line shows the final fit which includes the response functions from GEANT4 simulation [green (light) solid curves] and the additional exponential background plotted as dashed red line.

low-energy tail is due to single and double escape as well as the Compton events which remain after the Compton suppression treatment. The line shape obtained with the simulation was tested successfully in the case of ${ }^{22} \mathrm{O}$ [3]. The fitting of the broad structure in the ${ }^{25} \mathrm{~F}$ spectrum was made using the deduced $\gamma$ line shape and an exponential background, yielding $\gamma$ rays with energies of 3090(75), 3440(50), 3830(60), and $4210(80) \mathrm{keV}$ as shown in Fig. 1. These energies are in agreement with the values deduced after reevaluation of the spectra obtained in the single step fragmentation [18].

For the level scheme construction, $\gamma \gamma$-coincidence matrices were created. The most intense $1720 \mathrm{keV} \gamma$ line was found to be in coincidence with itself, as well as with the $750 \mathrm{keV}$ line. Furthermore, the $750 \mathrm{keV}$ transition is in mutual coincidence with a $3440 \mathrm{keV} \gamma$ ray as it can be seen in Fig. 2. In the analysis of the coincidence spectra the same line shapes and exponential background were assumed as for the single spectra.

The level scheme of ${ }^{25} \mathrm{~F}$ was established by using $\gamma \gamma$ coincidence matrices as well as energy and intensity balances. The energy and uncertainty of the excited states given below were determined taking into account the energies and uncertainties of all the $\gamma$ rays connected to a given state via the fitting procedure of the RADWARE package [21].

The coincidence relation of the $1720 \mathrm{keV} \gamma$ line with itself suggests two $\simeq 1720 \mathrm{keV} \gamma$ rays in cascade, establishing stats at about 1720 and $3400 \mathrm{keV}$. As this energy overlaps with that of the $3440(50) \mathrm{keV}$ transition within the experimental uncertainties, a level is proposed at $3440(21) \mathrm{keV}$. The $\gamma$ line of $750 \mathrm{keV}$ was chosen to feed the $3440 \mathrm{keV}$ level since it was in coincidence with both $\gamma$ rays of 1720 and $3440 \mathrm{keV}$. The sum 1720(15) + 1720(15) + 750(30) $\simeq 4190(60) \mathrm{keV}$ overlaps with the energy of the $4210(80) \mathrm{keV} \gamma$ ray establishing a state at $4195(35) \mathrm{keV}$. The remaining transitions at 3090(75) and 3830(60) keV, which were not observed in coincidence with any transitions, were placed to directly feed the ground state, establishing levels at the corresponding energies. A weak 2140(30) keV line was observed in the single step fragmentation reaction [18], the intensity of which was enhanced in the multiplicity 2 spectrum, suggesting that it was in coincidence with another transition. Energetically such a

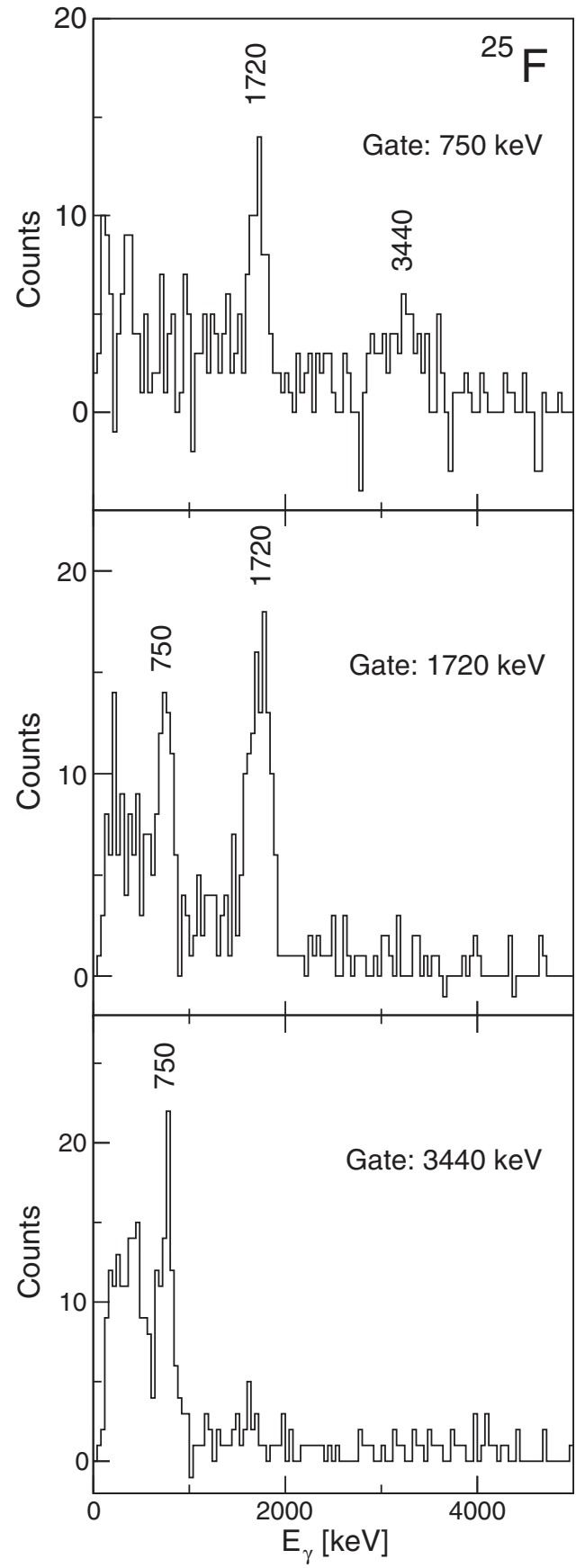

FIG. 2. Spectra of ${ }^{25} \mathrm{~F}$ from $\gamma \gamma$ coincidence using the 750, 1720, and $3440 \mathrm{keV}$ transitions as gates.

transition could connect the 1720 and $3830 \mathrm{keV}$ states. In the single step reaction the $3830 \mathrm{keV}$ line was the strongest, which explains why this transition was below the observation level in the present work.

The relative intensities of the 750(30), 3090(75), 3440(50), $3830(60)$, and $4210(80) \mathrm{keV}$ transitions were determined on the basis of energy-dependent $\gamma$-ray efficiencies. The situation was more complex for the unresolved $1720 \mathrm{keV}$ doublet, since the intensity of its members could not be derived directly. First the intensity ratio of the higher-lying member of the $1720 \mathrm{keV}$ doublet and of the $3440 \mathrm{keV} \gamma$ ray deexciting from 


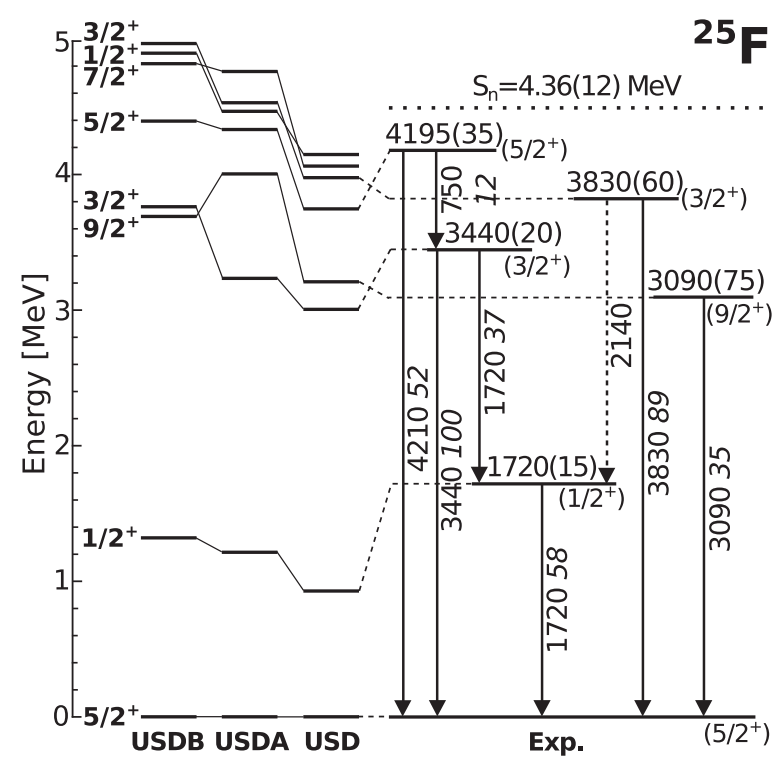

FIG. 3. Proposed level scheme of ${ }^{25} \mathrm{~F}$ compared to the shell model calculations performed using the USD, USDA, and USDB interactions. Energies are given along the transitions as well as their relative intensities in italics. The uncertainties of the relative intensities are below $20 \%$. The $2140 \mathrm{keV}$ line has been adopted from Ref. [18].

the 3440(20) $\mathrm{keV}$ state was derived in a spectrum gated with the $\gamma$ ray of $750 \mathrm{keV}$. From this ratio the intensity of the higherlying member of the $1720 \mathrm{keV}$ doublet was deduced using the corresponding peak areas in the single spectrum. The intensity of the lower-lying member of the doublet was obtained by subtracting the intensity of the higher lying component from the integral of the whole $1720 \mathrm{keV}$ intensity. The resulting intensities of the $\gamma$ transitions are presented in Fig. 3.

\section{DISCUSSION}

The established level scheme of ${ }^{25} \mathrm{~F}$ (Fig. 3) is compared to the results of shell-model calculations using effective interactions like the USD [22], USDA, and USDB [23] interactions defined for the $1 s 0 d$ valence space. From a naive singleparticle picture we expect that the ground state of ${ }^{25} \mathrm{~F}$ has spin and parity $J^{\pi}=5 / 2^{+}$. It corresponds to the filling of the $d_{5 / 2}$ single proton orbit above the ${ }^{24} \mathrm{O}$ core.

The first excited state at $1720(15) \mathrm{keV}$ is supposed to correspond to the $J^{\pi}=1 / 2^{+}$state. In the simple singleparticle picture, the $1 / 2^{+}$state can be interpreted as a proton excitation to the $1 s_{1 / 2}$ orbit. All other excited states, predicted above $3 \mathrm{MeV}$ in the $s d$ space, originate from the coupling of a proton single-particle state to excitations of the ${ }^{24} \mathrm{O}$ core in addition to the proton $d_{3 / 2}$ excitation. In addition, negative parity intruder or cluster states arising from a wider model space may also be present.

Considering that the state at $3440 \mathrm{keV}$ decays both to the ground state of $J^{\pi}=\left(5 / 2^{+}\right)$and the first excited $J^{\pi}=\left(1 / 2^{+}\right)$ state, a tentative $J^{\pi}=\left(3 / 2^{+}\right)$assignment can be given to this state. Similarly, the $4195 \mathrm{keV}$ state decays to the $J^{\pi}=\left(5 / 2^{+}\right)$ ground state and to the $J^{\pi}=\left(3 / 2^{+}\right)$excited state, but not to the $J^{\pi}=\left(1 / 2^{+}\right)$state, suggesting a $J^{\pi}=5 / 2^{+}$assignment for this state. Although, the selection rules allow for spin $3 / 2$ or $7 / 2$, too, the missing transition to the $\left(1 / 2^{+}\right)$state makes the spin $3 / 2$ assignment less probable, while the spin $7 / 2$ assignment is questioned by observation of the $750 \mathrm{keV}$ transition, since a $750 \mathrm{keV} \mathrm{E2}$ transition hardly could compete with an $4.2 \mathrm{MeV} M 1$ one. With the above spin assignment the $\gamma$ branching ratios are also in a qualitative agreement with the predictions of the $s d$ shell model. While comparable intensities are expected for the transitions from the $J^{\pi}=3 / 2_{1}^{+}$state to the $J^{\pi}=5 / 2_{1}^{+}$and $J^{\pi}=1 / 2_{1}^{+}$ones, the $M 1$ transition from the $J^{\pi}=5 / 2_{2}^{+}$to the $J^{\pi}=5 / 2_{1}^{+}$state is strongly hindered. This latter transition could only be observed since the $E_{\gamma}^{3}$ energy factor gives an enhancement of a factor of about 200.

All the shell-model interactions predict a $J^{\pi}=9 / 2_{1}^{+}$state below the $J^{\pi}=5 / 2_{2}^{+}$level. A good candidate for this $J^{\pi}=$ $9 / 2_{1}^{+}$level is the level derived at $3090(75) \mathrm{keV}$. On the basis of shell model calculations and the fact that it directly decays to the ground state, the $3830 \mathrm{keV}$ state's spin-parity can be limited to $1 / 2^{+}$to $9 / 2^{+}$. Considering that the $2140(30) \mathrm{keV}$ line observed in the single step reaction can also be assigned to the decay of the $3830 \mathrm{keV}$ state, a spin $\left(3 / 2^{+}\right)$might be assigned to this state. The branching ratios deduced from the single step reaction are consistent with the branching ratios calculated in the shell model for the $3 / 2_{2}^{+}$state.

In Fig. 3, we can see that rather strong deviations can be found between the different versions of the shell-model interactions. The USD interaction fits reasonably well with the experimental data, while the USDA and USDB interactions stretch the energy spectrum too much. Assuming the above tentative spin assignments, the higher energy group of the experimental states lies about $500 \mathrm{keV}$ below the predicted ones. This energy difference is similar to what was observed in ${ }^{24} \mathrm{O}$. Since most of these states originate from the coupling of the proton single-particle states to the $2^{+}$and $1^{+}$states of ${ }^{24} \mathrm{O}$ the deviation might come from the inaccurate description of the ${ }^{24} \mathrm{O}$ core excitation. In this context we mention that these states of ${ }^{24} \mathrm{O}$ have a $v s_{1 / 2} d_{3 / 2}$ dominant configuration, where the $1 s_{1 / 2}$ neutron is excited to the unbound $0 d_{3 / 2}$ orbit. In this connection it may be interesting to mention that to describe the new data-among others - the neutron $d_{3 / 2}$ single-particle energy was increased by 330 and $440 \mathrm{keV}$ in the USDA and USDB interactions, respectively, relative to the USD parametrization.

The unbound $d_{3 / 2}$ neutron may have an extended spatial distribution, which can result in a decrease of the interaction matrix elements in which the $d_{3 / 2}$ neutron is involved. Decreasing the $v s_{1 / 2} d_{3 / 2}$ interaction strength results in a decreased splitting of the $v s_{1 / 2} d_{3 / 2}$ doublet, yielding an increase in energy of the $2^{+}$state in ${ }^{24} \mathrm{O}$. Decrease of the $\pi d_{5 / 2} v d_{3 / 2}$ interaction causes the decrease of the splitting of the $\pi d_{5 / 2} \oplus 2^{+}$multiplet. As a result, both effects would worsen the agreement with experiment; thus the spatial extension of the unbound neutron $0 d_{3 / 2}$ orbit cannot explain the observed deviation. A possible explanation may be the coupling to continuum configurations, as proposed in [12].

A low-energy bound intruder state with $\operatorname{spin} J^{\pi}=1 / 2^{-}$ was predicted around the neutron separation energy at 4296 $\mathrm{keV}$ using the $0 p 1 s 0 d$ model space [10]. If bound, such a state would decay via an $E 1$ transition, predominantly to the $J^{\pi}=1 / 2^{+}$state. Experimentally, we do not observe any state 
with such decay property and energy, suggesting that the first intruder state in ${ }^{25} \mathrm{~F}$ is unbound in agreement with the results of Franck et al. [10].

We also performed microscopic [24] CC calculations (see [25] for a textbook presentation) and compared them with experimental results. The coupled-cluster method is ideally suited to calculate properties of closed-shell nuclei and their immediate neighbors. While the shell-model restricts the model space to all possible determinants constructed from a small set of single-particle orbitals around a closed shell core, the $\mathrm{CC}$ method restricts the number of particle-hole excitations allowed in the determinant basis but uses a large single-particle space. Such particle-hole correlations are summed to infinite order depending on the level of approximation. Coupledcluster theory is thus a nonperturbative method.

To describe ${ }^{25} \mathrm{~F}$, that has a proton attached to ${ }^{24} \mathrm{O}$, we use particle-attached equation-of-motion coupled-cluster theory (PA-EOM-CC) [26,27]. In PA-EOM-CC the ${ }^{25} \mathrm{~F}$ wave function is described by a linear expansion of $1 \mathrm{p}, 2 \mathrm{p}-1 \mathrm{~h}, 3 \mathrm{p}-2 \mathrm{~h}, \ldots$ excitations on top of the ground state of ${ }^{24} \mathrm{O}$. In our earlier applications, we truncated the expansion at the $2 \mathrm{p}-1 \mathrm{~h}$ level, which works particularly well for low-lying states that are dominated by $1 \mathrm{p}$ excitations from a closed-shell ground state [26-29].

In this work we are also interested in describing excited states in ${ }^{25} \mathrm{~F}$ that can be viewed as a proton attached to the $J^{\pi}=2^{+}$excited state in ${ }^{24} \mathrm{O}$. Clearly, to describe such states we need to go beyond the $2 \mathrm{p}-1 \mathrm{~h}$ truncation level. As a rule of thumb, the level of approximation should be one order more than what is considered important for the state under study. We therefore include $3 \mathrm{p}-2 \mathrm{~h}$ configurations as presented in Ref. [30], but as a first approach we only include the terms that determines the $3 \mathrm{p}-2 \mathrm{~h}$ amplitudes in the coupled-cluster singles and doubles (CCSD) approximation. The similarity transformation generated by solving the $\mathrm{CC}$ equations induce additional many-body terms in the Hamiltonian. Only diagrams that contain at most two-body parts of the Hamiltonian have been included at the $3 \mathrm{p}-2 \mathrm{~h}$ level, while all diagrams have been included up to the $2 \mathrm{p}-1 \mathrm{~h}$ level.

We use interactions from chiral effective field theory at two different orders. First we look at the newly optimized chiral interaction at next-to-next-to-leading order $\left(\mathrm{N}^{2} \mathrm{LO}\right)$ from Ref. [31]. Already at this order in the chiral expansion, three-body diagrams appear, but these have not been included here. This interaction resulted in an excellent agreement for both binding energies and selected excited states for oxygen isotopes. Second, we look at the chiral interaction at next-tonext-to-next-to-leading order $\left(\mathrm{N}^{3} \mathrm{LO}\right)$ from Ref. [32]. Here, the effects of three-nucleon forces are treated as density-dependent corrections to the nucleon-nucleon interaction by integrating one nucleon in the leading-order chiral three-nucleon force over the Fermi sphere with a Fermi momentum $k_{F}$ in symmetric nuclear matter [33]. We use the parameters already established in Ref. [29]. These parameters were used recently in a study of oxygen isotopes [29], and also for ${ }^{26} \mathrm{~F}$ in Ref. [2].

Due to the increased computational cost of including $3 \mathrm{p}-2 \mathrm{~h}$ configurations, the single-particle space is limited to a Hartree-Fock basis, built from $N=10$ major harmonic oscillator shells. This is not large enough for the total binding

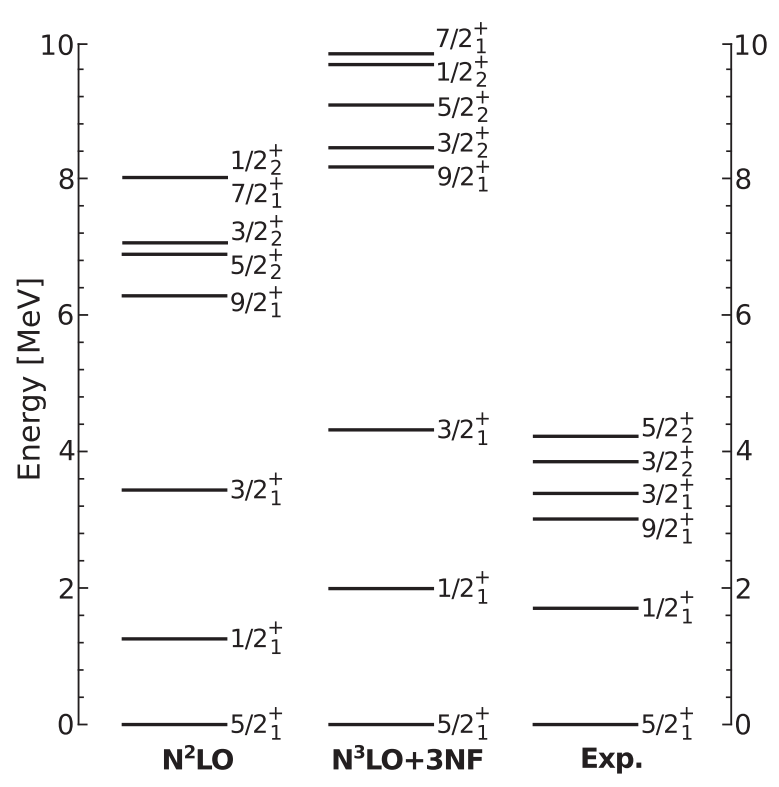

FIG. 4. Relative energies of ${ }^{25} \mathrm{~F}$ calculated using coupled-cluster theory with two different interactions compared with experiment. The label $\mathrm{N}^{2} \mathrm{LO}$ refers to the optimized chiral interaction at third order [31], while the interaction labeled $\mathrm{N}^{3} \mathrm{LO}+3 \mathrm{NF}$ is the chiral interaction of Ref. [32] where the effect of three nucleon forces are treated as a density-dependent nucleon-nucleon force (see text for details).

energies to be converged, but the relative energies exhibit a much faster convergence as function of the number of shells. The $\mathrm{N}^{2} \mathrm{LO}$ interaction is rather soft, meaning that the relative energies are practically converged at this level. On the other hand our results using the $\mathrm{N}^{3} \mathrm{LO}$ interaction exhibits a slower convergence rate, meaning that a larger model space is needed.

In Fig. 4 we plot the relative energies of selected positive parity states in ${ }^{25} \mathrm{~F}$ calculated with the two different interactions, as well as experimental energies. The first excited $J^{\pi}=1 / 2^{+}$and $J^{\pi}=3 / 2^{+}$states are reproduced reasonably well by both interactions, but the remaining states are at too high excitation energies as compared to the data. This deviation might suggest that for these states, the current level of approximation is not good enough. Including $4 \mathrm{p}-3 \mathrm{~h}$ configurations, where the latter account for $3 p-3 h$ excitations of the ${ }^{24} \mathrm{O}$ reference state, may further reduce the relative energies by 2 to $4 \mathrm{MeV}$. This guess is based on our observation that the relative energies for the high-lying states changes by 10 to $20 \mathrm{MeV}$ by going from the $2 \mathrm{p}-1 \mathrm{~h}$ to the $3 \mathrm{p}-2 \mathrm{~h}$ truncation level. To get a better insight into which contributions are important, we examine here in more detail the structure of the calculated wave functions.

Table I lists the partial norms of the PA-EOM-CC wave functions calculated with the optimized $\mathrm{N}^{2} \mathrm{LO}$ interaction. They sum up the parts of the wave function in $1 p, 2 p-$ $1 \mathrm{~h}$, and $3 \mathrm{p}-2 \mathrm{~h}$ configurations where the sum of all partial norms is one. We list only the partial norms for this $\mathrm{N}^{2} \mathrm{LO}$ interaction since those obtained with the $\mathrm{N}^{3} \mathrm{LO}$ interaction with density dependent three-body force produce qualitatively similar results. 
TABLE I. Partial norms of wave functions using up to $3 \mathrm{p}-2 \mathrm{~h}$ amplitudes on top of the ${ }^{24} \mathrm{O}$ reference state. The interaction is the newly optimized chiral interaction at third order $\left(\mathrm{N}^{2} \mathrm{LO}\right)$ [31].

\begin{tabular}{lccc}
\hline \hline & $1 \mathrm{p}$ & $2 \mathrm{p}-1 \mathrm{~h}$ & $3 \mathrm{p}-2 \mathrm{~h}$ \\
\hline $5 / 2_{1}^{+}$ & 0.63 & 0.30 & 0.07 \\
$1 / 2_{1}^{+}$ & 0.56 & 0.36 & 0.08 \\
$9 / 2_{1}^{+}$ & 0.00 & 0.74 & 0.26 \\
$3 / 2_{1}^{+}$ & 0.47 & 0.42 & 0.11 \\
$3 / 2_{2}^{+}$ & 0.01 & 0.72 & 0.27 \\
$5 / 2_{2}^{+}$ & 0.01 & 0.73 & 0.26 \\
$1 / 2_{2}^{+}$ & 0.03 & 0.72 & 0.25 \\
$7 / 2_{1}^{+}$ & 0.00 & 0.73 & 0.27 \\
\hline \hline
\end{tabular}

We see that the three presumed single-particle states have a large (30-40\%) contribution from $1 \mathrm{p}-1 \mathrm{~h}$ excitations of the ${ }^{24} \mathrm{O}$ reference state. If these were predominantly single-particle states, we would expect a contribution of approximately $10 \%$ or less from $2 \mathrm{p}-1 \mathrm{~h}$ configurations. As it turns out, $2 \mathrm{p}-1 \mathrm{~h}$ configurations are not enough to describe these states and they are not converged before $3 \mathrm{p}-2 \mathrm{~h}$ configurations are included. We see that roughly $10 \%$ of the wave functions for these three states come from $3 \mathrm{p}-2 \mathrm{~h}$ configurations, which implies a good level of convergence at this order. This is consistent with the shell-model calculations performed for these states. For the $J^{\pi}=5 / 2^{+}$ground state, the different USD interactions discussed above give wave functions with a 70 to $80 \%$ overlap with single-particle configurations, whereas the corresponding coupled-cluster numbers are 60 to $70 \%$. For the first excited $J^{\pi}=1 / 2^{+}$and $J^{\pi}=3 / 2^{+}$states, the single-particle content of the wave functions has dropped significantly in both models.

For the three lowest lying positive parity states $J^{\pi}=5 / 2_{1}^{+}$, $J^{\pi}=3 / 2_{1}^{+}$, and $J^{\pi}=1 / 2_{1}^{+}$, we note that the admixture from $3 \mathrm{p}-2 \mathrm{~h}$ configurations does not exceed some $10 \%$, meaning that we can interpret these states mainly in terms of $1 \mathrm{p}$ and $2 \mathrm{p}-1 \mathrm{~h}$ configurations. The relative energies of these states are also well converged within the chosen set of configurations.

We notice also that for other positive parity states like the $J^{\pi}=9 / 2_{1}^{+}, J^{\pi}=3 / 2_{2}^{+}$, and $J^{\pi}=5 / 2_{2}^{+}$states, there are considerable admixtures from $3 \mathrm{p}-2 \mathrm{~h}$ and more complicated configurations. Since additional $4 \mathrm{p}-3 \mathrm{~h}$ configurations are expected to reduce the relative energies by 2 to $4 \mathrm{MeV}$, these changes are most likely larger than contributions that can be obtained from continuum effects. In Refs. [12,29] we found that the coupling to the continuum in ${ }^{24} \mathrm{O}$ gave a reduction in relative energy of 300 to $500 \mathrm{keV}$ for states close to the neutron separation energy, just the energy difference found between the experimental results and the USDA/B calculations. As suggested by Table I, a suitable description of the high energy spectrum of ${ }^{25} \mathrm{~F}$ would require the treatment of contributions above the (already large dimensionality) $3 \mathrm{p}-2 \mathrm{~h}$ configurations. As this treatment is beyond the present computational limitations, a discussion of the continuum effects on these states has to be deferred to a later work.

We have also studied negative parity states within the PA-EOM-CC framework. We find a $J^{\pi}=3 / 2^{-}$state (not shown in Fig. 4) at approximately $10 \mathrm{MeV}$. It is identified as a spurious $J^{\pi}=1^{-}$center-of-mass excitation [34] built on the $J^{\pi}=5 / 2^{+}$ground-state configuration and is therefore not considered a physical state [27,35]. At around $12 \mathrm{MeV}$ we find a $J^{\pi}=1 / 2^{-}$state that is consistent with a physical state, but a large $3 \mathrm{p}-2 \mathrm{~h}$ component suggests that it is not yet converged at this level of approximation.

\section{CONCLUSION}

We studied the structure of ${ }^{25} \mathrm{~F}$ by the use of the inbeam $\gamma$ spectroscopy technique from a double-step fragmentation reaction. Utilizing the $\gamma$-spectroscopic information, we constructed a level scheme for ${ }^{25} \mathrm{~F}$, including the states corresponding to the coupling to the core excitation of ${ }^{24} \mathrm{O}$. Shell-model calculations using parametrized interactions for the $1 s 0 d$ shell account for many of the general features of the energy spectrum, although a $500 \mathrm{keV}$ deviation from the experiment was found in the USDA/B calculations for the temporarily assigned states of the $\pi d_{5 / 2} \oplus 2^{+}$multiplet. All the states observed could be interpreted within the $s d$ shell model; no bound negative parity intruder or cluster state has been found.

We also performed coupled-cluster calculations using recent interaction models from effective field theory. These calculations resulted in a very good agreement with experiment for the low-lying $J^{\pi}=5 / 2^{+}, 1 / 2^{+}$, and $3 / 2^{+}$states. The analysis of the wave functions showed also a qualitatively similar picture in terms of single-particle states as the shellmodel calculations. For higher-lying states that could be assigned to the $\pi d_{5 / 2} \oplus 2^{+}$multiplet, our coupled-cluster calculations yielded excited states at too high excitation energies. An analysis of the structure of the wave functions indicated that correlations beyond the current truncation level might be necessary. In ${ }^{24} \mathrm{O}$ we found that the coupling to the continuum gave a reduction in relative energy of 300 to $500 \mathrm{keV}$ for states close to the neutron separation energy, just the energy difference found between the experimental results and the USDA/B calculations. However, due to the large dimensionalities introduced by $3 \mathrm{p}-2 \mathrm{~h}$ configurations in this work, we were not able to provide a proper estimate of continuum effects in ${ }^{25} \mathrm{~F}$.

\section{ACKNOWLEDGMENTS}

This work was partly supported by the European Union's Seventh Framework Program under Grant Agreement No. 262010, by a grant of the Romanian National Authority for Scientific Research, CNCS-UEFISCDI, Project No. PN-II-IDPCE-2011-3-0487, and also by OTKA Contract No. K100835 and NN104543, PICS(IN2P3) 1171, INTAS 00-00463, GACR 202-04791, and RFBR N96-02-17381a grants, the Bolyai János Foundation, and the TÁMOP-4.2.2/B-10/1-2010-0024 project. The TÁMOP project is cofinanced by the European Union and the European Social Fund. In addition, this work was partly supported by the Office of Nuclear Physics, U.S. Department of Energy (Oak Ridge National Laboratory) under No. DE-SC0008499 (NUCLEI SciDAC-3 Collaboration), and the Field Work Proposal ERKBP57 at the Oak Ridge National Laboratory. An award of computer time was provided by the Innovative and Novel Computational Impact on Theory and 
Experiment (INCITE) program. This research used resources of the Oak Ridge Leadership Computing Facility located in the Oak Ridge National Laboratory, which is supported by the Office of Science of the Department of Energy under Contract No. DE-AC05-00OR22725 and used computational resources of the National Center for Computational Sciences, the National Institute for Computational Sciences, and the Notur project in Norway. Support from the Research
Council of Norway under Contract No. ISP-Fysikk/216699 is acknowledged. B.A.B. acknowledges support from NSF Grant No. PHY-1068217. This research was partly realized in the frames of the TÁMOP 4.2.4. A/2-11-1-2012-0001 National Excellence Program elaborating and operating an inland student and researcher personal support system. The project was subsidized by the European Union and cofinanced by the European Social Fund.
[1] A. Ozawa, T. Kobayashi, T. Suzuki, K. Yoshida, and I. Tanihata, Phys. Rev. Lett. 84, 5493 (2000).

[2] A. Lepailleur et al., Phys. Rev. Lett. 110, 082502 (2013).

[3] M. Stanoiu et al., Phys. Rev. C 69, 034312 (2004).

[4] C. R. Hoffman et al., Phys. Lett. B 672, 17 (2009).

[5] K. Tshoo et al., Phys. Rev. Lett. 109, 022501 (2012).

[6] R. Kanungo et al., Phys. Rev. Lett. 102, 152501 (2009).

[7] C. R. Hoffman et al., Phys. Rev. Lett. 100, 152502 (2008).

[8] R. B. Firestone, Nucl. Data. Sheets 110, 1691 (2009).

[9] M. Kimura and N. Furutachi, Phys. Rev. C 83, 044304 (2011).

[10] N. Frank et al., Phys. Rev. C 84, 037302 (2011).

[11] J. K. Smith et al., Phys. Rev. C 86, 057302 (2012).

[12] K. Tsukiyama, M. Hjorth-Jensen, and G. Hagen, Phys. Rev. C. 80, 051301(R) (2009).

[13] A. Volya, Phys. Rev. C 79, 044308 (2009).

[14] M. Belleguic et al., Nuc. Phys. A 682, 136 (2001).

[15] F. Azaiez et al., Eur. Phys. J. A 15, 93 (2002).

[16] F. Azaiez et al., Nucl. Phys. A 704, 37 (2002).

[17] Z. Elekes et al., Phys. Lett. B 599, 17 (2004).

[18] Zs. Vajta et al., Acta Phys. Pol. B 44, 553 (2013).

[19] R. Anne, Nucl. Instrum. Methods B 126, 279 (1997).

[20] L. Bianchi et al., Nucl. Instrum. Methods A 276, 509 (1989).

[21] D. C. Radford, Nucl. Instrum. Methods A 361, 297 (1995).

[22] B. H. Wildenthal, Prog. Part. Nucl. Phys. 11, 5 (1984); B. A. Brown and B. H. Wildenthal, Annu. Rev. Nucl. Part. Sci. 38, 29 (1988).
[23] B. A. Brown and W. A. Richter, Phys. Rev. C 74, 034315 (2006).

[24] F. Coester, Nucl. Phys. 7, 421 (1958); F. Coester and H. Kummel, ibid. 17, 477 (1960); J. Čížek, J. Chem. Phys. 45, 4256 (1966); J. Čížek and J. Paldus, Int. J. Quant. Chem. 5, 359 (1971).

[25] I. Shavitt and R. J. Bartlett, Many-Body Methods in Chemistry and Physics (Cambridge University Press, Cambridge, 2009).

[26] J. R. Gour, P. Piecuch, M. Hjorth-Jensen, M. Wloch, and D. J. Dean, Phys. Rev. C 74, 024310 (2006).

[27] G. Hagen, T. Papenbrock, D. J. Dean, and M. Hjorth-Jensen, Phys. Rev. C 82, 034330 (2010).

[28] G. Hagen, T. Papenbrock, and M. Hjorth-Jensen, Phys. Rev. Lett. 104, 182501 (2010).

[29] G. Hagen, M. Hjorth-Jensen, G. R. Jansen, R. Machleidt, and T. Papenbrock, Phys. Rev. Lett. 108, 242501 (2012).

[30] M. Musiał and R. J. Bartlett, J. Chem. Phys. 119, 1901 (2003)

[31] A. Ekstrom et al., Phys. Rev. Lett. 110, 192502 (2013).

[32] D. R. Entem and R. Machleidt, Phys. Rev. C 68, 041001 (2003).

[33] J. W. Holt, N. Kaiser, and W. Weise, Phys. Rev. C 79, 054331 (2009); 81, 024002 (2010).

[34] G. R. Jansen, Phys. Rev. C 88, 024305 (2013).

[35] G. Hagen, T. Papenbrock, and D. J. Dean, Phys. Rev. Lett. 103, 062503 (2009). 